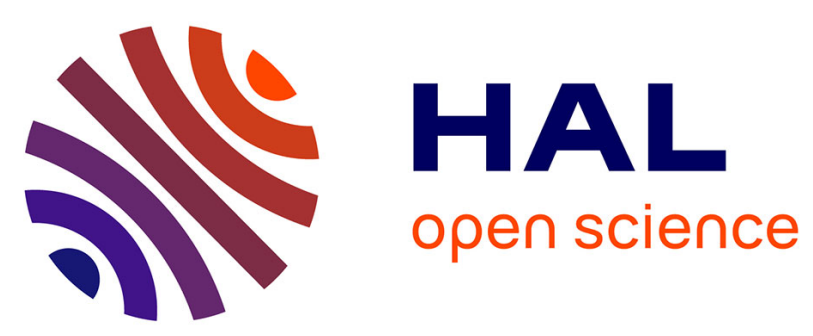

\title{
Self-Assembly of Magnetically-Functionalized Molecular Motors and Microtubules into Active Gels
}

Wei-An Wang, Marina Garcia-Jove Navarro, Zoher Gueroui

\section{To cite this version:}

Wei-An Wang, Marina Garcia-Jove Navarro, Zoher Gueroui. Self-Assembly of MagneticallyFunctionalized Molecular Motors and Microtubules into Active Gels. Soft Matter, 2019, 15 (44), pp.9111-9119. 10.1039/C9SM01227C . hal-02415651

\section{HAL Id: hal-02415651 \\ https://hal.sorbonne-universite.fr/hal-02415651}

Submitted on 17 Dec 2019

HAL is a multi-disciplinary open access archive for the deposit and dissemination of scientific research documents, whether they are published or not. The documents may come from teaching and research institutions in France or abroad, or from public or private research centers.
L'archive ouverte pluridisciplinaire HAL, est destinée au dépôt et à la diffusion de documents scientifiques de niveau recherche, publiés ou non, émanant des établissements d'enseignement et de recherche français ou étrangers, des laboratoires publics ou privés. 


\section{Self-Assembly of Magnetically-Functionalized Molecular Motors and Microtubules into Active Gels}

Wei-An Wang ${ }^{1}$, Marina Garcia-Jove Navarro ${ }^{1}$, Zoher Gueroui ${ }^{1}{ }^{*}$

1- PASTEUR, Department of Chemistry, École Normale Supérieure, PSL University, Sorbonne Université, CNRS, 75005 Paris, France.

*Correspondence: zoher.gueroui@ens.fr 


\begin{abstract}
The diversity of functions achieved by living cells result from the collective behavior of biological components that interact through multiple scales in time and space. The cytoskeleton constitutes one canonical system forming dynamic organizations when interacting with molecular motors. These materials constitute a state of active matter that exhibit out-ofequilibrium behavior with oriented order in the presence of energy. However, such active materials are highly dependent on the intrinsic properties of their constituents (fibers, molecular motors, energy), which render difficult to control their behavior. Being able to manipulate directly the constitutive elements of the active gel could provide additional control parameters. Here, we report a strategy to functionalize and manipulate active microtubule-based structures upon magnetic actuation. We engineered the protein nanocage ferritins as magnetic labels targeting molecular motors (Eg5 kinesin motors). We first mixed theses magnetic motors with individual microtubules, allowing their manipulation. In order to generate a magneticresponsive gel, we then mix the magnetic motors with active microtubule-based structures and characterize their dynamic behavior. We found that the magnetic forces applied on magnetic motors slowdown the dynamic of microtubule structures as well as constrain their rotation. Our result highlights how genetically encoded magnetic elements, behaving as magnetic actuators, could perturb active gels.
\end{abstract}




\section{Introduction}

The organization of living cells results from the collective behavior of biomolecules(1). Recent progresses in the field of cell biology allow to understand some of the general principles that can account for the generation of multiscale assemblies in cells, spanning from the nanometer to few tens of micrometers(2,3). In eukaryotic cells, the cytoskeleton fibers participate in building intracellular order, contribute to the mechanical integrity of the cell, as they power several essential functions such as cell migration or cell division(4-6). Several studies have demonstrated that biomolecule self-organization can be exploited to produce artificial structures and novel materials having emergent properties(7-11). For instance, the design of DNA origami allowed the construction of 2D and 3D assemblies serving as scaffolds to position molecules or nanoparticles with nanometer resolution(12,13). M13 filamentous bacteriophages have been engineered as scaffolds to display targeting ligands and nanoparticles(14). These strategies lead to bio-assemblies that can be robustly produced on demand and with potentially a high throughput(15). However, such nano-structures form stable assemblies and generally operate at thermodynamic equilibrium, which restrain their use as static scaffolds. In contrast, cytoskeleton structures made of microtubules and actin filaments can produce dynamic assemblies that are constantly remodeled by molecular motors, in order to generate macroscopic order and emergent behavior, such as long range motion or contractile flow(16-18). Active gels constituted of microtubules and molecular motors are remarkable examples of dynamic self-organized structures very well suited to study the minimal set of elements leading to spatial patterning and spontaneous motion(19,20). Pioneer experiments have shown that a mixture of tubulins and multimeric kinesin motors was sufficient to account for the generation of microtubule asters and vortexes(19,21). Besides, at high concentration, such elements percolate to form a network that undergo complex flows and hydrodynamic instabilities $(20,22)$. The dynamical properties of active mater is highly dependent on the intrinsic properties of the biomolecules, their concentration, the amount of energy, the geometrical boundary and confinement $(23,24)$. Being able to control their dynamic behavior using external stimuli could provide an additional control parameter. Previous studies have reported the action of external fields on microtubule structures. Microtubule alignment and orientation has been achieved by hydrodynamic forces $(25,26)$, or by magnetic fields applied during tubulin polymerization(27,28). A recent study reported how microtubule-based active nematic gels, in contact with a thermotropic liquid crystal, can have their orientation dictated by a magnetic field(29). As magnetic interactions can be contactless, remote controlled and can 
deeply penetrate into thick materials, it could be advantageous to manipulate directly the constitutive elements of the active gel, as it has been shown for other biomolecules(30-32). In our study, we developed a strategy to label kinesin motors with magnetic nanoparticles in order to perturb the dynamical properties of a microtubule active gel. We used mineralized and genetically modified ferritins as magnetic labels to interact with the kinesin Eg5, using a chemical-inducible dimerization strategy (Figure 1). By mixing such magnetic molecular motors with stabilized microtubules, we formed individual magnetic assemblies that respond to magnetic forces. Next we generated an active magnetic gel by mixing the magnetic molecular motors with a preformed active material, based on kinesin clusters and microtubule filaments. Finally, we examined how the magnetic field can alter the dynamical properties of the microtubule-based active gel. 


\section{Results}

\section{Functionalization of kinesin Eg5 to magnetic ferritins}

In order to functionalize the kinesin Eg5 to magnetic ferritins, we used a chemicalinducible dimerization strategy(33). Briefly, we genetically fused FKBP (FK506 binding protein) and mCherry fluorescent protein to the N-terminal domain of the Pyrococcus furiosus ferritin monomer to generate FKBP-mCherry-ferritin nanocages. We applied in vitro biomineralization to direct the synthesis of iron oxide nanoparticles inside the apoferritin cavities, by loading iron into purified recombinant FKBP-mCherry-ferritins (Methods). To form the final Eg5-kinesin-ferritin complexes (Eg5-Ftn), we mixed in a 1:1 ratio the FKBPmCherry-ferritins $(0.25 \mu \mathrm{M})$ and Eg5-eGFP-FRB $(0.25 \mu \mathrm{M})$ in presence of rapamycin $(10 \mu \mathrm{M})$. This strategy led to obtain in average a distribution of stoichiometry of Eg5:Ftn, with an average of $1: 1$ coexisting with $0: 1$ and 2:1 (Eg5:Ftn). We next validated that the application of a gradient of magnetic field $\left(10^{4} \mathrm{~T} . \mathrm{m}^{-1}\right)$ was sufficient to attract free-diffusing Eg5-Ftn towards the magnet (Figure S1a, Supplementary Video S1).

Next, microtubules were formed by incubating purified tubulin $(10 \mu \mathrm{M})$ in a polymerization buffer at $37^{\circ} \mathrm{C}$ for 30 minutes (Methods). Once assembled, the microtubules were stabilized by taxol and mixed to Eg5-Ftn complexes to form Eg5-kinesin-ferritinmicrotubule assemblies (Eg5-Ftn-MT) (Figure 2a). Observations by fluorescence microscopy showed that Eg5-Ftn colocalized with the microtubules and covered the fibers homogenously (Figure 2b). The average length of the Eg5-Ftn-MT was about $5 \pm 1.4 \mu \mathrm{m}(\mathrm{n}=100)$ (Figure 2c, left, and Figure S2), which corresponded to the length of the microtubules before functionalization $(4 \pm 0.9 \mu \mathrm{m}, \mathrm{n}=100$, Figure $2 \mathrm{c}$, right). MT morphology after functionalization was almost identical in term of length and brightness, but we cannot rule out also the presence of MT bundles (Figure S2). Moreover, we found that increasing the amount of Eg5-eGFP-FRB compared to FKBP-mCherry-ferritins, in a 2:1 ratio in average, induced the cross-linking of microtubules and the formation of a meshwork (Figure S1b). 


\section{Magnetic manipulation of motor-microtubule structures}

We next examined the possibility to spatially manipulate motor-microtubule structures (Eg5-Ftn-MT). We encapsulated a water-based solution containing the Eg5-Ftn-MT structures in oil droplets with a confinement diameter of about 100-200 $\mu \mathrm{m}$ (one to four microtubule structures per droplet, Figure 3a). In absence of magnetic field, microtubule structures fluctuated within the droplet volume because of thermal agitation. To apply a gradient of magnetic field, we positioned at the vicinity of the droplet a permanent magnet coupled with a magnetic tip (Figure 3a). We observed that the majority of the microtubule fibers displayed a motion towards the magnetic tip and could rotate during their displacement (Figure 3b). We tracked individual microtubule trajectories and measured an average speed of about $230 \pm 120$ $n m \cdot s^{-1}(n=30$, Figure 3c). The variability of the microtubule speed can be explained by the heterogeneity of the number of Eg5-Ftn per microtubule as well as by the microtubule length dispersion or polymorphism (Figure 3d).

Altogether, our data show that the biomineralized Eg5-Ftn can be used to manipulate microtubule structures with a magnetic field.

\section{Assembly of a magnetic active gel}

Active microtubule gels display very rich spatiotemporal patterns depending on the concentration of the constituents and the depleting agents, the motor multivalency and the energy amount(20). To establish our active gel-based assay, we built on previous studies that examined the dynamics of microtubule bundles powered by the activity of multimeric kinesins(20). Briefly, we generated active microtubule gels using $60 \mu \mathrm{M}$ of microtubules, stabilized with the non-hydrolysable nucleotide analogue GMPCPP. Microtubule bundles were formed by adding a depleting agent, polyethylene glycol (PEG), which induced attractive interactions through depletion forces. We assembled multimeric motors, "Kin-clusters", by mixing kinesins fused to biotins to tetrameric streptavidins in an average stoichiometry of two kinesin-biotins for one streptavidin. Under these conditions, the Kin-clusters consisted in a mixture from 1 to 4 kinesins per streptavidin, allowing the motor complexes to simultaneously bind and move along microtubules, inducing inter-filament sliding forces. We next confined the active gel in water-in-oil droplets of a size of about 100-200 $\mu \mathrm{m}$ (Methods). Time-lapse fluorescence microscopy revealed that microtubule bundles were highly dynamic and formed an array of aligned fibers that globally rotate in random fashion within the droplet (Figure S3, Supplementary Video S2). 
In order to provide magnetic properties to the active material, we mixed the Eg5-Ftn complexes (stoichiometry 2:1, $100 \mathrm{nM}$ final) to the preformed microtubule active gel. Eg5-Ftn complexes appeared as discrete fluorescent dots along microtubule fibers, suggesting their incorporation into the gel (Figure $4 \mathrm{a}$ ). In those conditions, the microtubule gel lost its dynamic properties and behaved as static at the time scale of few tens of minutes. The gel morphology was also changed and the microtubule bundles appeared thicker and shorter in length. The static behavior of the microtubule gel observed may be caused by the larger number of cross-linking nodes induced by Eg5-Ftns. By their multivalency, they could counterbalance the inter-filament sliding required to power the motion of the active gel. In accordance with this picture, by decreasing the concentration of Kin-clusters from $250 \mathrm{nM}$ to $70 \mathrm{nM}$, despite the presence of Eg5-Ftn (100 nM:50 nM), the dynamic properties of the active gel were for the most part restored (Figure 4b, Supplementary Video S3). Indeed, the dynamics of the Eg5-functionalized active gel did not perfectly reproduce the spatiotemporal patterns observed in Figure S3a, but the long-range microtubule alignment and rotational movement were still observed. On the other hand, the replacement of the whole fraction of Kin-clusters by Eg5-Ftn led mainly to a static microtubule gel (only $15 \%$ of the gel that were formed had a dynamical behavior). These latest observations could be explained by the difference of processivity between the Eg5-Ftn and the Kin-clusters $\left(81 \mathrm{~nm} . \mathrm{s}^{-1}\right.$ versus $733 \mathrm{~nm} . \mathrm{s}^{-1}$ respectively $\left.(34,35)\right)$.

Collectively, our results illustrate that the microtubule active gel properties are very sensitive to the concentration of Kin-cluster motors.

\section{Effect of a magnetic field on microtubule active gel}

We next examined the dynamic properties of the microtubule active gel (70 nM of Kincluster and $100 \mu \mathrm{M}$ of Eg5-Ftn) in presence of a magnetic tip positioned next to the boundary of the confined droplets, as previously performed. Time-lapse microscopy observations showed that the dynamic behavior of the active gel was changed upon magnetic field application. The orientation of the microtubule bundles composing the active gel was constrained and their rotation was drastically decreased (Figure 4c, Supplementary Video S4). This strong reduction of the microtubule rotation was confirmed by kymograph analysis: microtubule bundles remained statically orientated within the timescale of about $10 \mathrm{~min}$, whereas in the absence of magnetic field, kymograph showed microtubule bundles randomly rotating (Figure 4c, Supplementary Video S5).

To further characterize the impact of the magnetic field, we assessed the nature of the motion of the fluorescent Eg5-Ftn. We tracked fluorescent Eg5-Ftn (Figure 5a) and computed 
the Mean Squared Displacement (hereafter called MSD) from the obtained trajectories (Figure $5 \mathrm{~b}, \mathrm{n}=50)$. In the absence of magnetic field, the log of the MSD displayed a linear dependence as function of time, with a slope of about 1.7, indicating a super-diffusive motion (Figure 5c). We measured an effective speed of $200 \mathrm{~nm} \cdot \mathrm{s}^{-1}$ (Figure 5e), which is larger than the reported value of Eg5 speed $\left(80 \mathrm{~nm} \cdot \mathrm{s}^{-1}\right)$. This can be explained by the contribution of the microtubule translational motion occurring during the gel remodeling. Furthermore, in presence of the magnetic field, the MSD of the Eg5-Ftn were found to be 5-fold smaller than the MSD without the magnetic field (Figure 5d). The reduced mobility of the Eg5-Ftn was also confirmed by measuring the average speed of the complexes (4 fold smaller in average), as shown in Figure 5e. Yet, the temporal evolution of the MSD was unaffected (slope $\sim 1.7$, Figure 5c), suggesting that the super-diffusive motion of the Eg5-Ftn remained unchanged. In addition, the Eg5-Ftn complexes displayed shorter trajectories under magnetic forces (Figure 5a).

Altogether, these data suggest that the magnetic forces oppose to the motor activity of the Eg5-Ftn, which corroborates with the constrained motion characterizing the microtubule gel.

\section{Discussion}

Using engineered ferritins as magnetic labels targeting Eg5 kinesin motors, we examined how magnetic forces impact the dynamic of an active microtubule gel made of stabilized microtubule bundles stirred by multivalent kinesin motors. We found that the mixture of Eg5-Ftn with the active gel changed its morphology and preclude its dynamics. Nevertheless, the tuning of the constituents allowed to recover most of the dynamic features. The dynamic of active MT gel is often explained by considering the sliding of filaments powered by multivalent-kinesins generating sliding forces on anti-parallel microtubules. Our system is more complex and contains also an ensemble of Eg5-Ftn that can further produce pushing and braking forces depending on the MT polarity. It has been reported that the forces generated by an ensemble of Eg5 motors cross-linking two microtubules differ in the parallel and anti-parallel of microtubules. In this picture, Eg5-Ftn could for instance inhibit motor motion in parallel cross-linked MTs. Interestingly, the application of a magnetic field slowed down the overall dynamic of the gel and constrained its capacity to rotate in a confined environment. This was confirmed by monitoring the Eg5-Ftn motion that showed an overall reduction of the MSD, mean velocity, and trajectory length.

How the active gel slowdown under magnetic field actuation can be explained? When applying a magnetic field gradient of about $10^{4} \mathrm{~T} / \mathrm{m}$, we estimate that we generated a force of 
about $1 f N$ on single mineralized ferritin(33). This force is not sufficient to inhibit Eg5-Ftn processivity, since the stalling forces is about $p N$, or to favor the unbinding of molecular motors from microtubule filaments. This was confirmed by the observation that Eg5-Ftn complexes remained attached to microtubule fibers (Figure S3b), as well as by the absence of free fluorescent Eg5-Ftn accumulation towards the magnetic tip. Conversely, one hypothesis is that the collective magnetic forces applied on Eg5-Ftn could counterbalance the inter-filament sliding forces that shear microtubule fibers and, eventually, constrain their rotation. In our experimental condition, we estimate that about a thousand of Eg5-Ftn complexes can bind to on a $5 \mu \mathrm{m}$-long MT fibre, which could collectively result in generating $p N$ forces. Furthermore, the magnetic forces slowdown Eg5-Ftn motion without changing their mode of diffusion, as indicated by their anomalous diffusion index (1.7). Thus, depending on MT polarity, magnetic forces can modulate pushing and braking forces on MT network. However, despite the overall active gel slowdown, the approach did not enable us to control the manipulation of the orientation of the active nematic, as it has previously been achieved using thermotropic liquid crystals (29).

Altogether, our work underlies the possibility to directly manipulate constitutive elements of an active gel by magnetic means. The present approach has the advantage of using genetically encoded constitutive elements that behave as magnetic actuators, which opens new venues towards the control and production of active biomaterials or in the context of probing intracellular structures(36). 


\section{Materials and methods}

\section{Reagents}

Buffers powders, ATP, H2O2, FeSO4, Sodium citrate, DTT and mineral oil (M5904) were purchased from Sigma-Aldrich (St Louis, MO). Tubulins, labelled with Rhodamin, Cy5 or with FITC, were ordered from Cytoskeleton Inc. (Denver, CO). Rapamycin was purchased from Calbiochem. Jurkat. All reagents for buffer preparation were purchased from Sigma-Aldrich.

\section{Expression and purification of recombinant proteins}

The plasmid for Escherichia coli expression of the wild type ferritin (Pyrococcus furiosus) is a gift from W.R Hagen(37), the Eg5-kinesin-eGFP-FRB molecular motor is a gift from W.O Hancock(34). All plasmids were cloned in pET28 plasmid (FRB-mCherry-ferritin, Eg5-GFPFRB). The recombinant proteins were produced in Rosetta (DE3) pLysS cells (Merck Millipore) by addition of IPTG (Sigma) followed by overnight expression. The purification of the recombinant proteins was realized using standard his-tagged protein purification protocols.

\section{Biomineralisation of ferritins}

The iron oxides nanoparticles were prepared as previously described(38). Briefly, purified FKBP-mCherry-ferritins $(24 \mu \mathrm{M})$ were mineralized at $65^{\circ} \mathrm{C}$ in $5 \mathrm{mM}$ HEPES solution. Solution of Ammonium Iron (II) Sulfate hexahydrate was used as iron source. All solutions were degassed with argon and the $\mathrm{pH}$ was maintained dynamically at 8.5 using an automatic titrator (718,Titrator, Methrohm). Fe(II) was added to attain a loading factor of $3000 \mathrm{Fe}$ per protein cage. The $\mathrm{Fe}(\mathrm{II})$ and $\mathrm{H} 2 \mathrm{O} 2(3: 1)$ were added simultaneously at constant rate of $15 \mu \mathrm{L} . \mathrm{min}^{-1}$ using a syringe pump ( $\mathrm{Kd}$ scientific). The reaction was considered finished 3 hours after addition of all iron and oxidant solutions. The mineralized sample was dialyzed overnight against $5 \mathrm{mM}$ HEPES solutions.

\section{Microtubule polymerization for single microtubules functionalization}

Tubulin and Cy5-labelled tubulin were purchased from tebu-bio. Tubulin and Cy5-labelled tubulin were copolymerized with $3 \%$ of labelled dimers. The polymerization mixture consisted of $1 \mu \mathrm{M}$ of Cy5-labelled tubulin, $30 \mu \mathrm{M}$ of unlabeled tubulin, 5\% glycerol, $1 \mathrm{mM} \mathrm{GTP,} 1 \mathrm{mM}$ DTT, and BRB80 buffer to a final volume of $10 \mu \mathrm{L}$. The suspension was incubated for $30 \mathrm{~min}$ at $37^{\circ} \mathrm{C}$, and microtubules were stabilized with $30 \mu \mathrm{M}$ Taxol.

\section{Single microtubules functionalization with Eg5-Ftn complexes}


First, Eg5-Kinesin-eGFP-FRB-FKBP-mCherry-Ferritin complexes (Eg5-Ftn) were assembled by mixing $1.25 \mu \mathrm{L}$ of $2 \mu \mathrm{M}$ Eg5-Kinesin-eGFP-FRB with $2.5 \mu \mathrm{L}$ of $1 \mu \mathrm{M}$ FKBP-mCherryFerritin in the presence of $0.2 \mu \mathrm{L}$ of $500 \mu \mathrm{M}$ Rapamycin. The mixture was incubated for 5 minutes on ice. Then $0.2 \mu \mathrm{L}$ of GTP $(2 \mathrm{mM}), 0.66 \mu \mathrm{L}$ of Taxol $(300 \mu \mathrm{M})$ and $0.5 \mu \mathrm{L}$ of microtubule polymerization mix $(30 \mu \mathrm{M})$ were added to the mixture and diluted with BRB80 buffer for a final volume of $10 \mu \mathrm{L}$. The mixture was incubated at room temperature for 10 minutes.

\section{Water-in-oil droplet formation}

Functionalized microtubule structures were encapsulated in droplets via water-in-oil emulsion process. Mineral oil contains a biocompatible block copolymer in order to stabilize emulsion and facilitate observations. The mixture was then gently sheared by pipetting the solution up and down for a few seconds to generate extract-in-oil droplets. The mechanical dispersion of the biphasic solution formed micrometer-sized water-in-oil droplets within a few seconds. The emulsion was observed under the fluorescent microscope. The droplets presenting had a diameter comprised between 100 and $200 \mu \mathrm{m}$.

\section{Magnetic manipulation of microtubule structures}

A magnetic tip was elongated from a guitar string leading to a radius of curvature of $25 \mu \mathrm{m}$. The tip was then adapted on the N-S axis of an NdFeB permanent magnet ( $3 \mathrm{~mm}$ length) and placed next to water-in-oil droplets using a manual micromanipulator (Narishige). With this system, the gradient of magnetic field can reach $10^{4} \mathrm{~T} . \mathrm{m}^{-1}$ at the vicinity of the tip.

\section{Microtubule polymerization for microtubule active gel}

Tubulin was copolymerized with fluorescently labelled tubulin, producing microtubules with $3 \%$ of labelled monomers. The polymerization mixture consisted of $4.5 \mathrm{ml}\left(13.2 \mathrm{mg} \cdot \mathrm{ml}^{-1}\right)$ unlabeled tubulin, $0.9 \mathrm{ml}\left(7.4 \mathrm{mg} \cdot \mathrm{ml}^{-1}\right)$ Alexa-647-labelled tubulin, $1.4 \mathrm{ml}$ glycerol (to $15 \%$ of final volume), $0.56 \mathrm{ml}$ GMPCPP (10 mM) (39), $0.47 \mathrm{ml}$ dithiothreitol (DTT, $20 \mathrm{mM}$ ), and BRB80 buffer to a final volume of $9.4 \mathrm{ml}$ (for a final tubulin concentration of $6.8 \mathrm{mg} \cdot \mathrm{ml}^{-1}$ ). The suspension was incubated for $30 \mathrm{~min}$ at $37{ }^{\circ} \mathrm{C}$, and subsequently diluted to $6 \mathrm{mg} \cdot \mathrm{ml}^{-1}$ with BRB80. This resulted in an average microtubule length of $4 \mu \mathrm{m}$.

\section{Assembly of the microtubule-based active gel}


The formation of the active microtubule gels were assembled as previously described(20). Briefly, biotinylated Eg5-kinesin motor protein and tetrameric streptavidin (Invitrogen; 434301) aqueous suspensions were incubated on ice for $30 \mathrm{~min}$ at the specific stoichiometric ratio 2:1 to obtain kinesin-streptavidin motor clusters (Kin-clusters). Microtubules were mixed with the motor clusters that acted as cross-linkers, and with ATP (Sigma; A2383) that drove the activity of the gel. The aqueous dispersion contained a non-adsorbing polymeric agent (PEG, $20 \mathrm{kDa}$; Sigma; 95172) that promoted the formation of filament bundles through depletion (Fig. S1). Several antioxidant components were also included in the solution to avoid protein denaturation, and to minimize photobleaching during characterization by means of fluorescence microscopy. Microtubule active gels were encapsulated and observed with the same protocol as described above.

\section{Imaging and Data Analysis}

Fluorescence imaging of microtubule was performed using an IX81 (Olympus) and X60 oil objective (PlanApo, Na 1.42), equipped with an EM-CDD camera (electron multiplying CCD, C9100-02, Hamamatsu, Corporation) and a LED system of illumination (Spectra X, Lumencor). Microscope settings and functions were controlled using Simple PCI software (Hamamatsu). Image analysis was performed using Simple PCI and ImageJ. 


\section{Author's contributions}

WA.W and Z.G conceived and analysed the experiments. WA.W carried out the experiments. M.GJN contributed to the initial experiments and in designing the protein constructs. WA.W and Z.G wrote the manuscript and all authors were involved in revising it critically for important intellectual content.

\section{Acknowledgements}

We thank William O. Hancock for the gift of the Kin5-GFP-FRB plasmid. We acknowledge F.Guyot, J.M. Guigner, and the members of the Biophysical Chemistry group of the department of Chemistry of ENS for fruitful discussions. WA.W was supported by a "Ministère de la Recherche" predoctoral fellowship. M.G.J.N was supported by FRM (ING20150532742). This work was supported by the CNRS, the Ecole Normale Supérieure and the ANR (ANRNanoheater).

\section{Competing financial interests}

The authors declare no competing financial interests. 


\section{References}

1. Karsenti E. Self-organization in cell biology: a brief history. Nat Rev Mol Cell Biol [Internet]. 2008/02/23 . 2008;9(3):255-62. Available from: http://www.ncbi.nlm.nih.gov/entrez/query.fcgi?cmd=Retrieve $\& d b=P u b M e$ $\mathrm{d} \&$ dopt $=$ Citation\&list_uids $=18292780$

2. Misteli T. The concept of self-organization in cellular architecture. J Cell Biol. 2001/10/18. 2001;155(2):181-5.

3. Kirschner M, Mitchison T. Beyond self-assembly: from microtubules to morphogenesis. Cell [Internet]. 1986;45(3):329-42. Available from: http://www.ncbi.nlm.nih.gov/entrez/query.fcgi?cmd=Retrieve \&db=PubMe $\mathrm{d} \&$ dopt $=$ Citation\&list_uids $=3516413$

4. Pollard TD, Cooper JA. Actin, a Central Player in Cell Shape and Movement. Science (80- ). 2009;326:1208-12.

5. Li R, Gundersen GG. Beyond polymer polarity: how the cytoskeleton builds a polarized cell. Nat Rev Mol Cell Biol [Internet]. 2008/10/24. 2008;9(11):860-73. Available from: http://www.ncbi.nlm.nih.gov/entrez/query.fcgi?cmd=Retrieve $\& d b=P u b M e$ $\mathrm{d} \&$ dopt $=$ Citation\&list_uids $=18946475$

6. Letort G, Ennomani H, Gressin L, Théry M, Blanchoin L. Dynamic reorganization of the actin cytoskeleton. F1000Research [Internet]. 2015; Available from: http://f1000research.com/articles/4-940/v1

7. Hess H. Self-assembly driven by molecular motors. Soft Matter. 2006;2(8):669.

8. Seeman NC. DNA in a material world. Nature [Internet]. 2003 Jan 23 [cited 2015 Jan 31];421(6921):427-31. Available from: http://dx.doi.org/10.1038/nature01406

9. Leduc PR, Robinson DN. Using lessons from cellular and molecular structures for future materials. Adv Mater. 2007;19:3761-70. 
10. Galland R, Leduc P, Guérin C, Peyrade D, Blanchoin L, Théry M. Fabrication of three-dimensional electrical connections by means of directed actin self-organization. Nat Mater. 2013;12:416-421.

11. Aoyama S, Shimoike M, Hiratsuka Y. Self-organized optical device driven by motor proteins. Proc Natl Acad Sci U S A [Internet]. 2013/09/26. Available from:

http://www.ncbi.nlm.nih.gov/entrez/query.fcgi?cmd=Retrieve $\& d b=P u b M e$ $\mathrm{d} \&$ dopt $=$ Citation\&list_uids $=24065817$

12. Schreiber R, Do J, Roller E-M, Zhang T, Schüller VJ, Nickels PC, et al. Hierarchical assembly of metal nanoparticles, quantum dots and organic dyes using DNA origami scaffolds. Nat Nanotechnol. 2014;9:74-78.

13. Gür FN, Schwarz FW, Ye J, Diez S, Schmidt TL. Toward Self-Assembled Plasmonic Devices: High-Yield Arrangement of Gold Nanoparticles on DNA Origami Templates. ACS Nano. 2016;10,5:5374-5382.

14. Ghosh D, Lee Y, Thomas S, Kohli AG, Yun DS, Belcher AM, et al. M13templated magnetic nanoparticles for targeted in vivo imaging of prostate cancer. Nat Nanotechnol. 2012;7:677-682.

15. Hung AM, Micheel CM, Bozano LD, Osterbur LW, Wallraff GM, Cha JN. Large-area spatially ordered arrays of gold nanoparticles directed by lithographically confined DNA origami. Nat Nanotechnol. 2010;5:121126.

16. Needleman D, Dogic Z. Active matter at the interface between materials science and cell biology. Nature Reviews Materials. 2017;2:17048.

17. Menzel AM. Tuned, driven, and active soft matter. Physics Reports. 2015.

18. Doostmohammadi A, Ignés-Mullol J, Yeomans JM, Sagués F. Active nematics. Nature Communications. 2018;9:3246.

19. Nedelec FJ, Surrey T, Maggs AC, Leibler S. Self-organization of microtubules and motors. Nature. 1997;389:305-8.

20. Sanchez T, Chen DTN, DeCamp SJ, Heymann M, Dogic Z. Spontaneous 
motion in hierarchically assembled active matter. Nature. 2012;491:431-4.

21. Surrey T, Nedelec F, Leibler S, Karsenti E. Physical properties determining self-organization of motors and microtubules. Science (80- ). 2001;292:1167-71.

22. Wu KT, Hishamunda JB, Chen DTN, DeCamp SJ, Chang YW, FernándezNieves A, et al. Transition from turbulent to coherent flows in confined three-dimensional active fluids. Science (80- ). 2017;355: eaal1979.

23. Henkin G, DeCamp SJ, Chen DTN, Sanchez T, Dogic Z. Tunable dynamics of microtubule-based active isotropic gels. Philos Trans R Soc A Math Phys Eng Sci. 2014;372: 20140142.

24. Kasza KE, Rowat AC, Liu J, Angelini TE, Brangwynne CP, Koenderink $\mathrm{GH}$, et al. The cell as a material. Current Opinion in Cell Biology. 2007;19:101-107.

25. Brown TB, Hancock WO. A Polarized Microtubule Array for KinesinPowered Nanoscale Assembly and Force Generation. Nano Lett. 2002;2(10):1131-5.

26. Prots I, Stracke R, Unger E, Böhm KJ. Isopolar microtubule arrays as a tool to determine motor protein directionality. Cell Biol Int. 2003;27:251253.

27. Glade N, Tabony J. Brief exposure to high magnetic fields determines microtubule self-organisation by reaction-diffusion processes. Biophys Chem. 2005;115(1):29-35.

28. Platt M, Muthukrishnan G, Hancock WO, Williams ME. Millimeter scale alignment of magnetic nanoparticle functionalized microtubules in magnetic fields. J Am Chem Soc. 2005;127(45):15686-7.

29. Guillamat P, Ignés-Mullol J, Sagués F. Control of active liquid crystals with a magnetic field. Proc Natl Acad Sci. 2016;113(20):5498-502.

30. Kashida S, Wang DO, Saito H, Gueroui Z. Nanoparticle-based local translation reveals mRNA as a translation-coupled scaffold with anchoring 
function. Proc Natl Acad Sci U S A. 2019; Available from:

https://doi.org/10.1073/pnas.1900310116

31. Hoffmann C, Mazari E, Lallet S, Le Borgne R, Marchi V, Gosse C, et al. Spatiotemporal control of microtubule nucleation and assembly using magnetic nanoparticles. Nat Nanotechnol [Internet]. 2013;8(3):199-205. Available from: http://www.ncbi.nlm.nih.gov/pubmed/23334169

32. Etoc F, Lisse D, Bellaiche Y, Piehler J, Coppey M, Dahan M. Subcellular control of Rac-GTPase signalling by magnetogenetic manipulation inside living cells. Nat Nano [Internet]. 2013;8:193-8. Available from: http://dx.doi.org/10.1038/nnano.2013.23

33. Ducasse R, Wang W-A, Navarro MG-J, Debons N, Colin A, Gautier J, et al. Programmed Self-Assembly of a Biochemical and Magnetic Scaffold to Trigger and Manipulate Microtubule Structures. Sci Rep. 2017;7: 11344.

34. Chen Y, Rolls MM, Hancock WO. An EB1-kinesin complex is sufficient to steer microtubule growth in vitro. Curr Biol. 2014;24:316-321.

35. Schnitzer MJ, Block SM. Kinesin hydrolyses one ATP per 8-nm step. Nature. 1997;388:386-390.

36. Tanimoto H, Sallé J, Dodin L, Minc N. Physical forces determining the persistency and centring precision of microtubule asters. Nat Phys. 2018; 14:848-854.

37. Matias PM, Tatur J, Carrondo MA, Hagen WR. Crystallization and preliminary X-ray characterization of a ferritin from the hyperthermophilic archaeon and anaerobe Pyrococcus furiosus. Acta Crystallogr Sect F Struct Biol Cryst Commun. 2005;61(5):503-6.

38. Uchida M, Flenniken ML, Allen M, Willits DA, Crowley BE, Brumfield $\mathrm{S}$, et al. Targeting of cancer cells with ferrimagnetic ferritin cage nanoparticles. J Am Chem Soc. 2006;128(51):16626-33.

39. Hyman A, Drechsel D, Kellogg D, Salser S, Sawin K, Steffen P, et al. Preparation of modified tubulins. Methods Enzym [Internet]. 1991/01/01. 
1991;196:478-85. Available from:

http://www.ncbi.nlm.nih.gov/entrez/query.fcgi?cmd=Retrieve $\& d b=P u b M e$ $\mathrm{d} \& \mathrm{dopt}=$ Citation\&list_uids $=2034137$ 


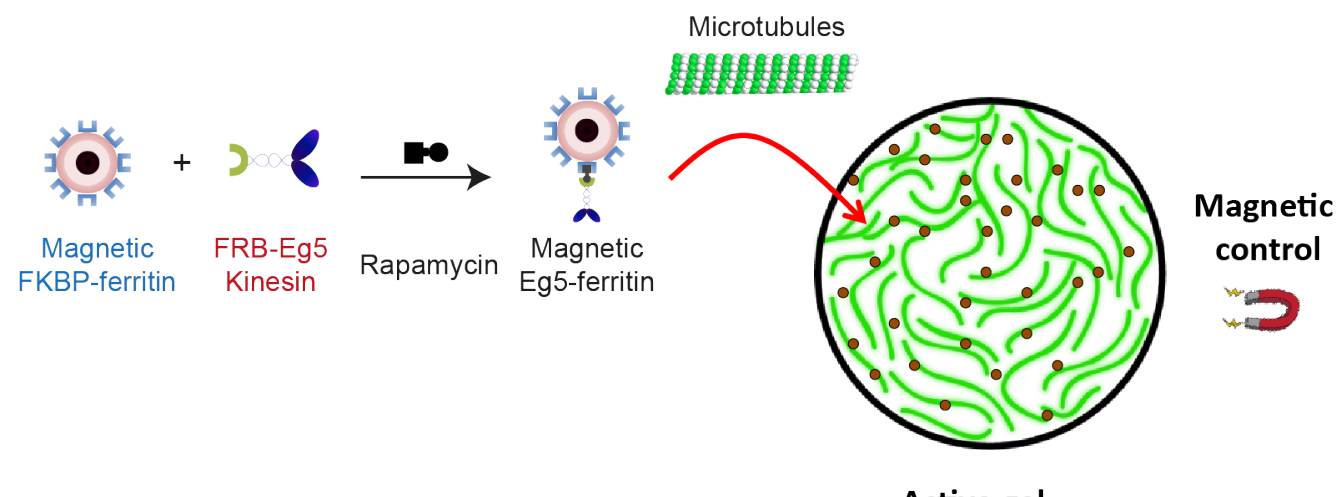

Active gel

Figure 1. Schematic of the functionalization of microtubules with magnetic molecular motors. The functionalization of the kinesin $\mathrm{Eg} 5$ to magnetic ferritins is mediated by the heterodimerization of FRB and FKBP upon rapamycin addition. Then, the magnetic motors are mixed with a preformed active microtubule gel. 
a

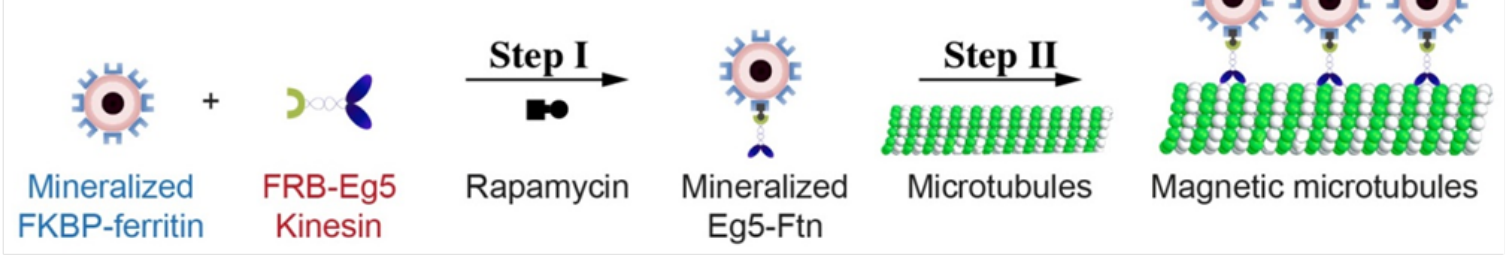

b Cy5-tubulin

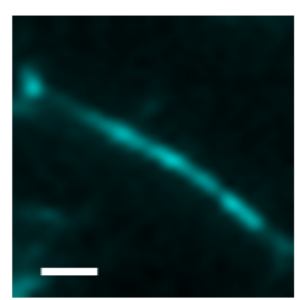

C

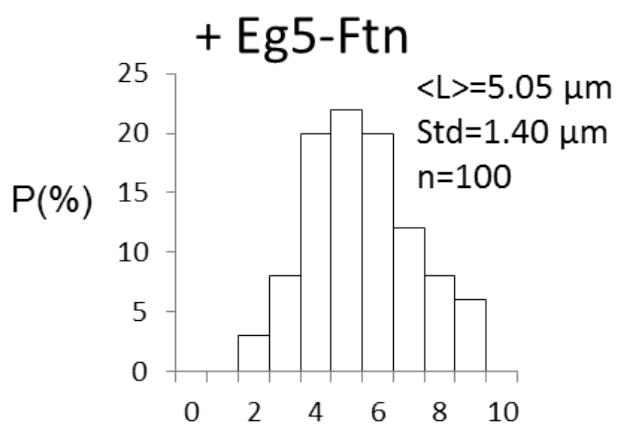

Microtubule length $(\mu \mathrm{m})$
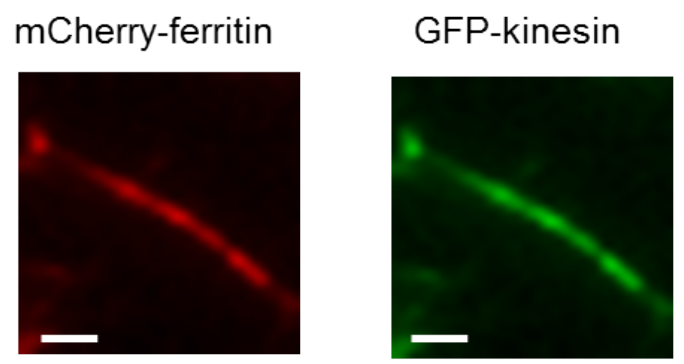

Merge
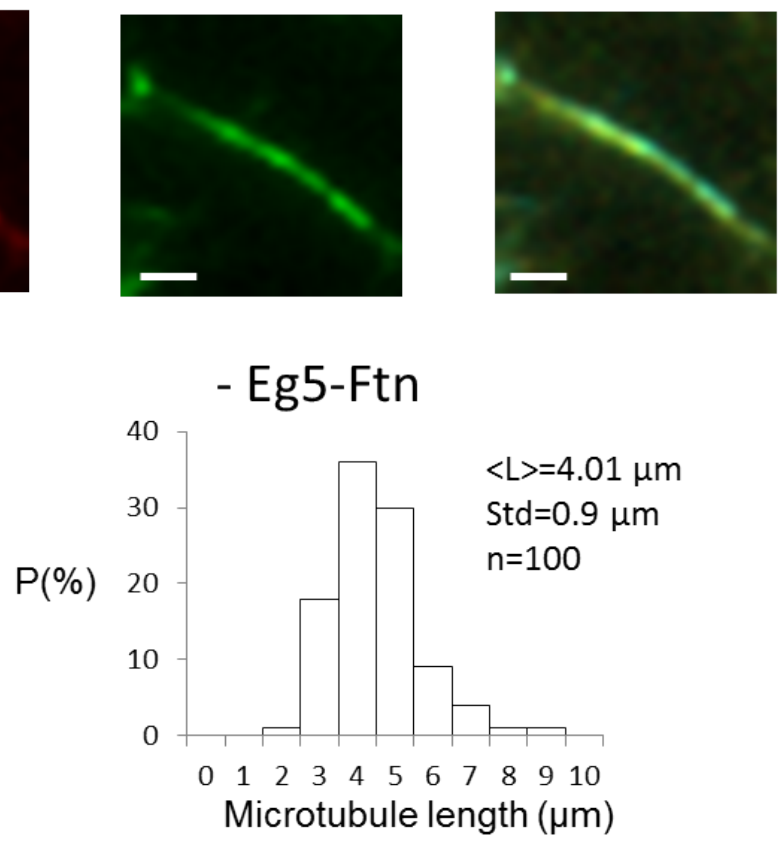

Figure 2. (a) Schematic of the functionalization of microtubules with magnetic Eg5-Ftn. Step I: The heterodimerization of FKBP-mCherry-ferritin with Eg5-Kinesin-eGFP-FRB in presence of rapamycin lead to the formation of Eg5-Ftn complexes. Step II: Microtubules functionalization by Eg5-Ftn. (b) Fluorescence microscopy showing the colocalization of Eg5-Ftn complexes with microtubules: Ratio 1:1 for FKBP-mCherry-ferritin and Eg5-Kinesin-eGFP-FRB. Scale bar $=200 \mathrm{~nm}$. (c) Length distribution of polymerized microtubules: Left, in the presence of Eg5-Ftn, microtubules have an average length of $5 \mu \mathrm{m}$ and a standard deviation of about $1.4 \mu \mathrm{m}$. Right, in the absence of Eg5-Ftn, microtubules have an average length of $4 \mu \mathrm{m}$ and a standard deviation of about $0.9 \mu \mathrm{m}$. 

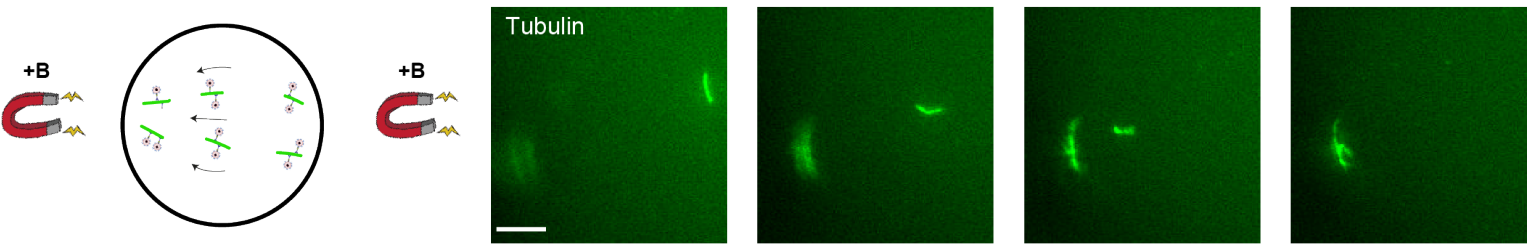

C
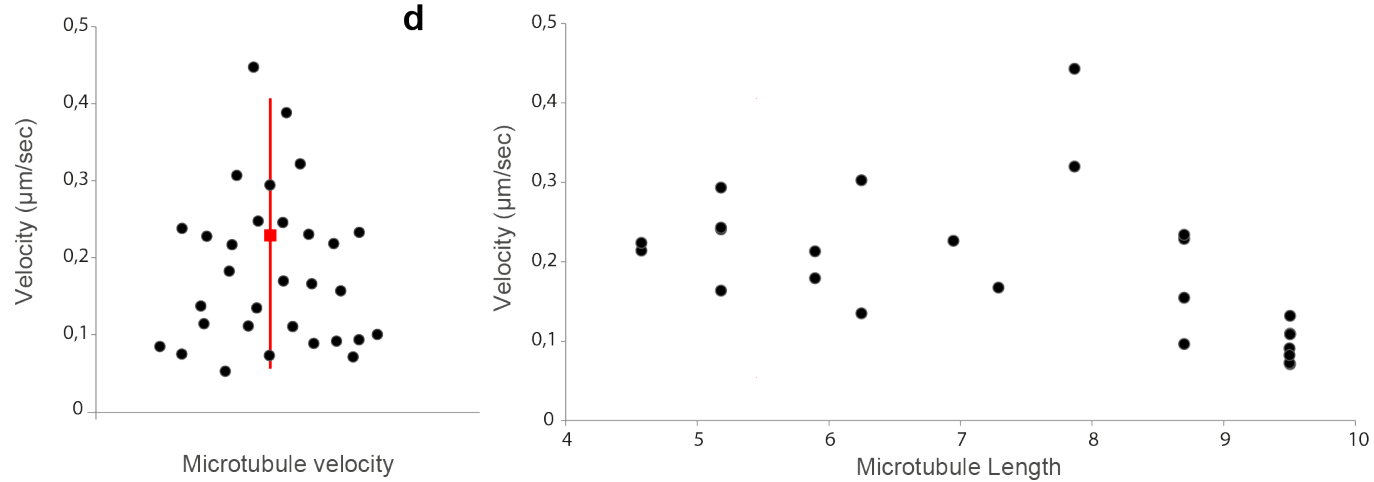

Figure 3. (a) Illustration of the magnetophoretic experiments performed on individual microtubule structures. (b) Time-lapse of the spatiotemporal dynamics of microtubule structures in the presence of a magnetic field $(+B)$. Scale bar $=10 \mu \mathrm{m}$. (c) Quantitative analysis of microtubules speed extracted from kymograph analysis. The microtubule average velocity is about $230 \mathrm{~nm} . \mathrm{s}^{-1}$ with a standard deviation of $120 \mathrm{~nm} \cdot \mathrm{s}^{-1}$. (d) Plot of the microtubule velocity as a function of microtubule length. 
a

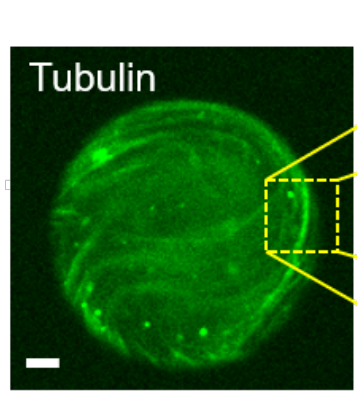

Merge

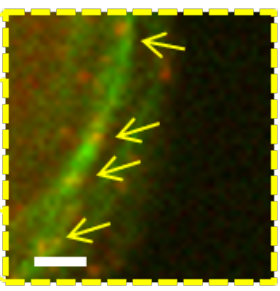

b
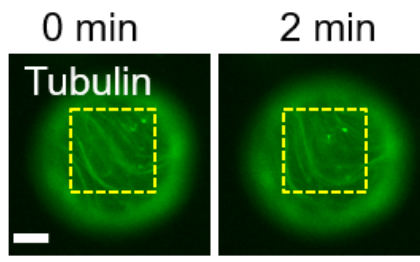

$4 \mathrm{~min}$
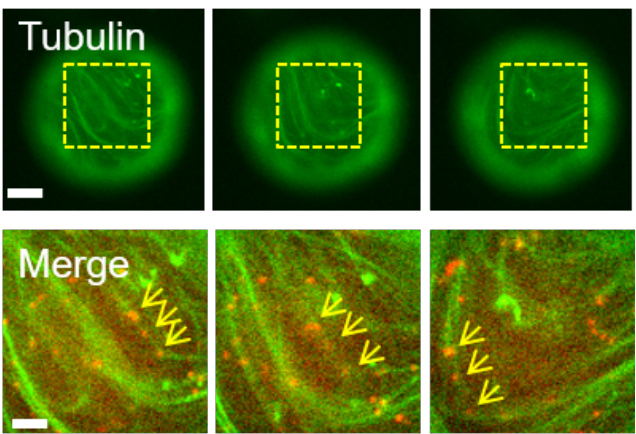

C

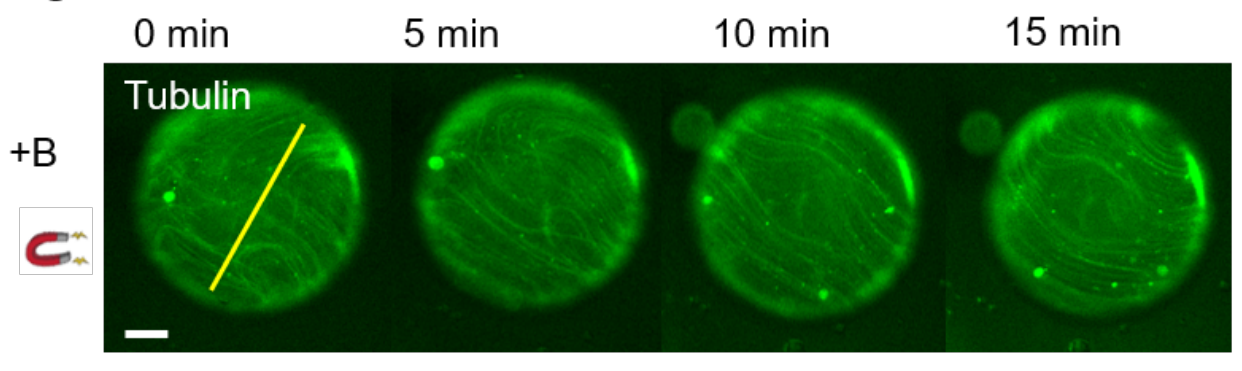

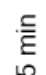

kymograph

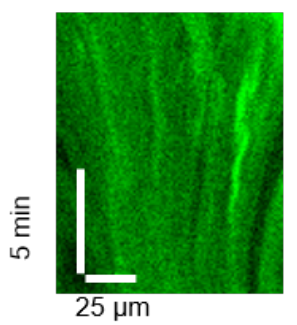

$$
0 \mathrm{~min}
$$

$5 \min$

$10 \min$

$15 \min$

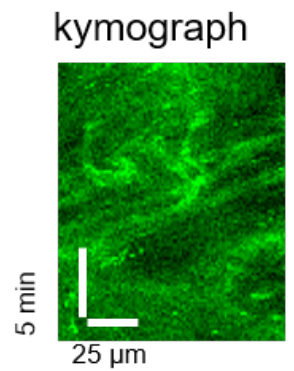

Figure 4. (a) Fluorescence imaging showing the colocalization of Eg5-Ftn (labelled with mCherry and indicated with yellow arrow) with microtubules (labelled with Cy5). Eg5-Ftn (red dots) colocalize with microtubules. Scale bar $=10 \mu \mathrm{m}$. Inset, scale bar $=2 \mu \mathrm{m}$ (b) Time-lapse of active microtubule gel (green) functionalized with magnetic Eg5-Ftn complexes (red) showing the dynamic properties of the active gel in the condition of $100 \mathrm{nM}$ Eg5-Ftn and $70 \mathrm{nM}$ Kin-cluster. (c) Representative time-points of the dynamic of a microtubule active gel (top) upon magnetic field actuation, and (bottom) in absence of magnetic field actuation. Scale bar $=10 \mu \mathrm{m}$. On the right: Kymographs obtained from the yellow dash lines. 

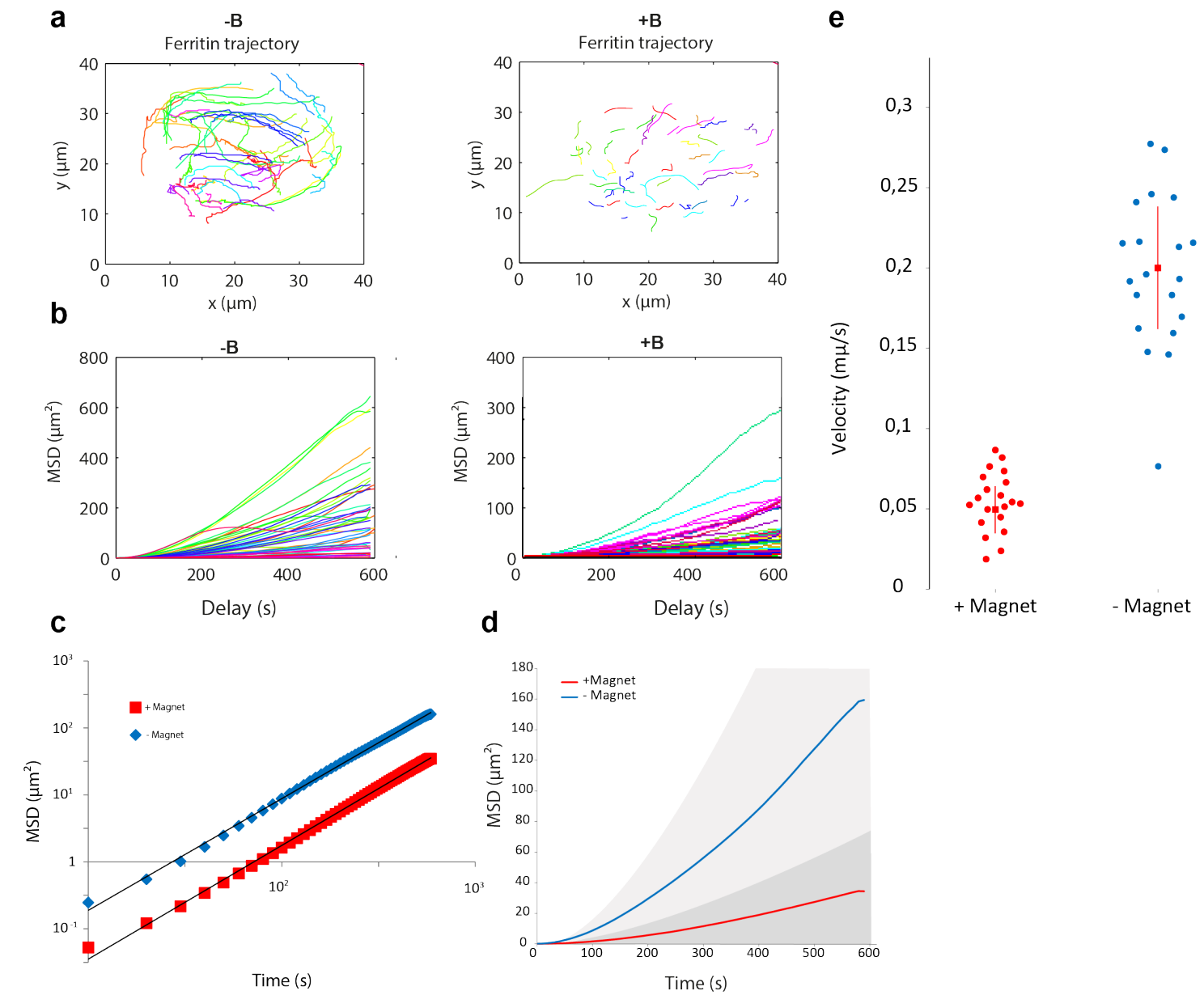

Figure 5. Tracking analysis of fluorescent Eg5-Ftn. (a) Tracking of Eg5-Ftn trajectories within droplets. (b) Mean Square Displacement (MSD) as a function of time for each individual trajectory in the absence of magnetic field actuation (left panel) and upon magnetic field actuation (right panel). (c) Log-log plot of the average MSD shown in (a). The $\alpha$ slope is 1.69 for red line and 1.67 for blue line. (d) Average of the MSD upon magnetic field actuation (red line) and in the absence of magnetic field actuation (blue line). Grey area: standard deviation of the individual MSD. (e) Quantification of the Eg5-Ftn velocity upon magnetic field actuation (red dots, $50 \pm 10 \mathrm{~nm} \cdot \mathrm{s}^{-1}$ ) and in the absence of magnetic field (blue dots, $\left.200 \pm 36 \mathrm{~nm} \cdot \mathrm{s}^{-1}\right)$. 


\section{Supplementary Figures}
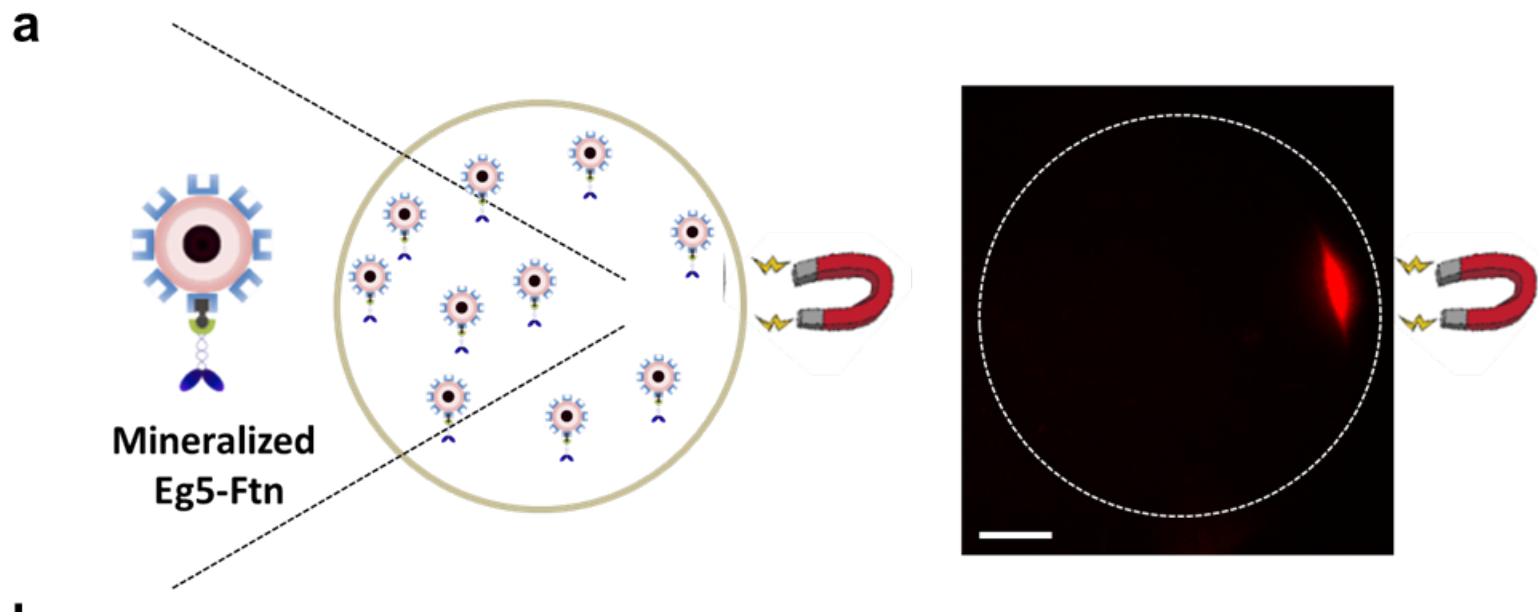

b
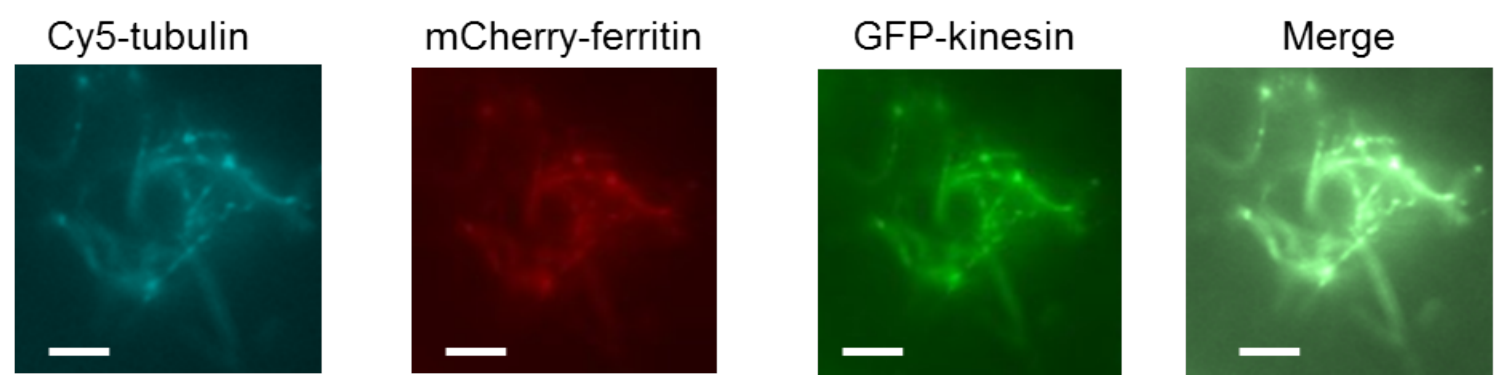

Figure S1 (a) Magnetic manipulation of Eg5-Ftn complexes. The Eg5-Ftns were labelled with mCherry. Magnetic forces were induced by a permanent magnet to attract of Eg5-Ftn complexes. Scale bar $=10$ $\mu \mathrm{m}$. (b) Fluorescence microscopy showing the colocalization of Eg5-Ftn complexes with microtubules: Ratio 1:2 for FKBP-mCherry-ferritin and Eg5-Kinesin-eGFP-FRB. Scale bar $=2 \mu \mathrm{m}$. 

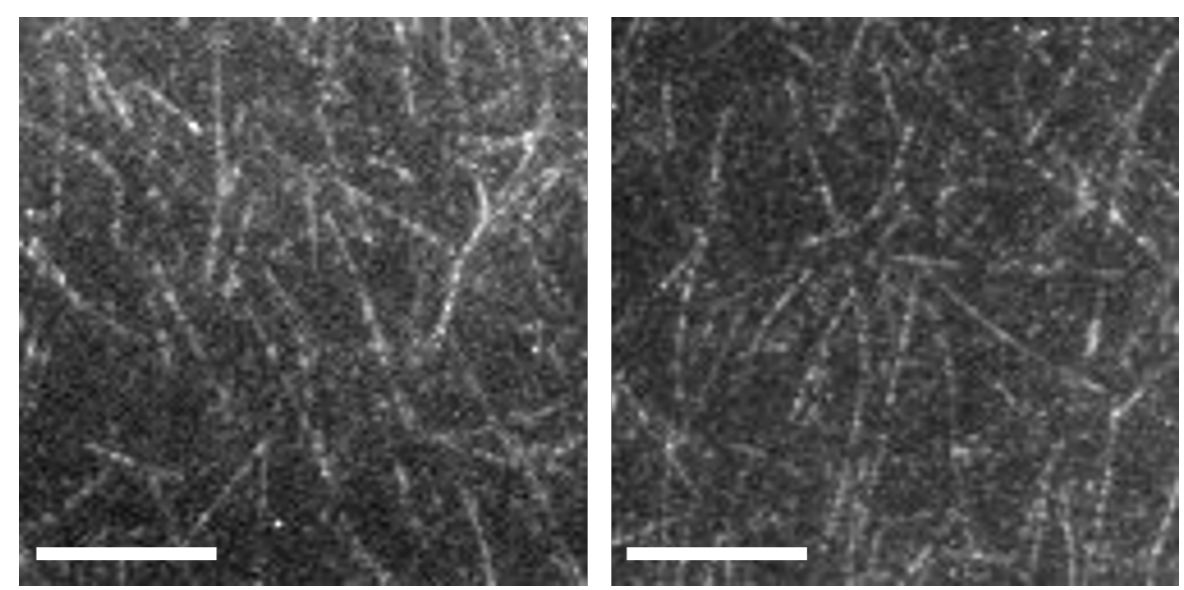

Figure S2 Observations by fluorescence microscopy of microtubule fibers before with (left panel) and after (right panel) being functionalized with Eg5-Ftn. Scale bar $=5 \mu \mathrm{m}$. 

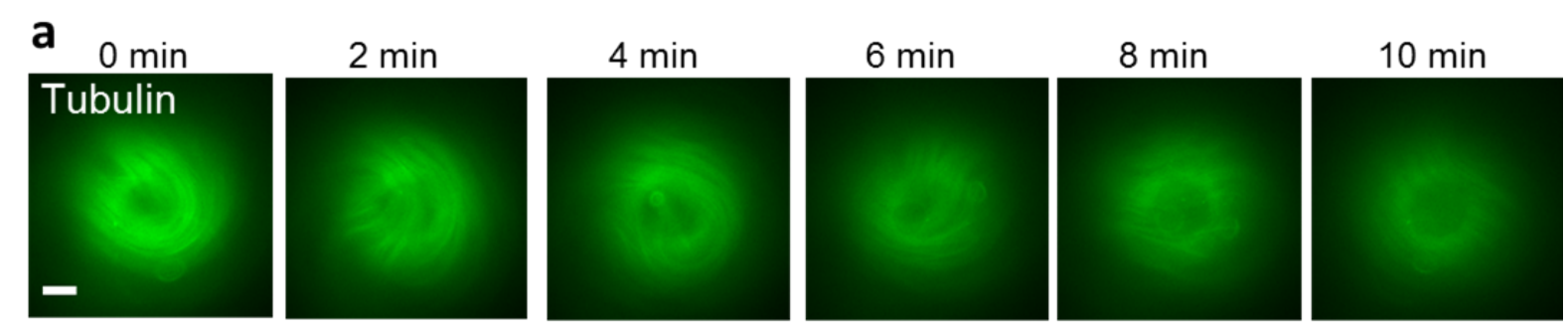

b

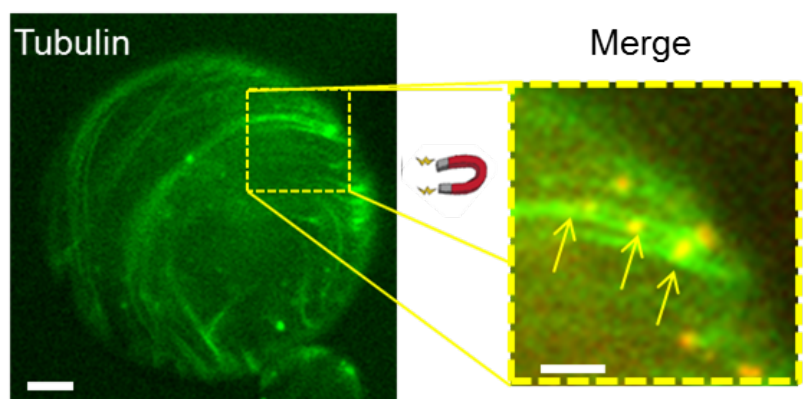

Figure S3 (a) Time-lapse fluorescence microscopy of active microtubule gel encapsulated within a droplet, illustrating the dynamic behavior of the microtubule networks. The concentration of Kin-cluster is $250 \mathrm{nM}$. Scale bar $=20 \mu \mathrm{m}$. (b) Colocalization of Eg5-Ftn (labelled with mCherry) with microtubules (labelled with Cy5-tubulin) upon magnetic field actuation. Scale bar $=10 \mu \mathrm{m}$ and $2 \mu \mathrm{m}$ in the zoom. 
Supplementary video S1: Magnetic attraction of Eg5-Ftn upon magnetic field application, corresponding to Figure S1a. Droplet size is $60 \mu \mathrm{m}$ and the video was recorded at $1 \mathrm{~s}$ intervals for $4 \mathrm{~min}$.

Supplementary video S2: Time-lapse of the dynamic of confined active microtubule gel, corresponding to Figure S2a. Droplet size is $100 \mu \mathrm{m}$ and the video was recorded at $5 \mathrm{~s}$ intervals for $10 \mathrm{~min}$.

Supplementary video S3: Time-lapse of the dynamic of confined active microtubule gel functionalized with magnetic Eg5-Ftn complexes, corresponding to Figure 4b. Droplet size is $75 \mu \mathrm{m}$ and the video was recorded at $5 \mathrm{~s}$ intervals for $5 \mathrm{~min}$.

Supplementary video S4: Time-lapse of the dynamic of confined active microtubule gel functionalized with magnetic Eg5-Ftn upon magnetic field actuation, corresponding to Figure $4 \mathrm{c}(+\mathrm{B})$. Droplet size is $60 \mu \mathrm{m}$ and the video was recorded at 10 s intervals for $15 \mathrm{~min}$.

Supplementary video S5: Time-lapse of the dynamic of confined active microtubule gel functionalized with magnetic Eg5-Ftn in absence of magnetic field (-B), corresponding to Figure 4c. Droplet size is $60 \mu \mathrm{m}$ and the video was recorded at 10 s intervals for $15 \mathrm{~min}$. 